\title{
Sum Rate Analysis of Multiple-Access Neuro-Spike Communication Channel with Dynamic Spiking Threshold
}

\author{
Tooba Khan ${ }^{\mathrm{a}, *}$, Ozgur B. Akan ${ }^{\mathrm{a}, \mathrm{b}}$ \\ ${ }^{a}$ Next-generation and Wireless Communications Laboratory (NWCL), Department of Electrical and Electronics Engineering, Koc University, \\ Istanbul 34450, Turkey \\ ${ }^{b}$ Internet of Everything (IoE) Group, Electrical Engineering Division, Department of Engineering, University of Cambridge, Cambridge CB3 \\ $O F A, U K$
}

\begin{abstract}
The information from outside world is encoded into spikes by the sensory neurons. These spikes are further propagated to different brain regions through various neural pathways. In the cortical region, each neuron receives inputs from multiple neurons that change its membrane potential. If the accumulated change in the membrane potential is more than a threshold value, a spike is generated. According to various studies in neuroscience, this spiking threshold adapts with time depending on the previous spike. This causes short-term changes in the neural responses giving rise to short-term plasticity. Therefore, in this paper, we analyze a multiple-input single-output (MISO) neuro-spike communication channel and study the effects of dynamic spiking threshold on mutual information and maximum achievable sum rate of the channel. Since spike generation consumes a generous portion of the metabolic energy provided to the brain, we further put metabolic constraint in calculating the mutual information and find a trade-off between maximum achievable sum rate and metabolic energy consumed. Moreover, we analyze three types of neurons present in the cortical region, i.e., Regular spiking, Intrinsic bursting and Fast spiking neurons. We aim to characterize these neurons in terms of encoding/transmission rates and energy expenditure. It will provide a guideline for the practical implementation of bio-inspired nanonetworks as well as for the development of ICT-based diagnosis and treatment techniques for neural diseases.
\end{abstract}

Keywords:

Neuro-spike Communication, Nanonetworks, Molecular Communications, MISO Neuro-spike Communication Channel, Channel Capacity, Metabolic Cost, Dynamic Threshold

\section{Introduction}

The networks of nano-sized machines, i.e., nanonetworks, find vast applications in health care, drug delivery and environmental monitoring while overcoming the functional limitations of the individual network entities due to their size [1, 2, 3]. Molecular Communications is a promising paradigm for realizing a new generation of nanoscale networks $[4,5,6,7]$. Therefore, extensive studies are being carried out to understand molecular communications by studying the natural processes. Consequently, neuronal signaling or neuro-spike communication is one of the most investigated natural phenomenon to study fundamentals of molecular communications channel. In [8], physical channel model of single-input single-output (SISO) neuro-spike communication is formulated. Three-dimensional propagation model of neurotransmitters in the synaptic cleft is proposed in [9]. Moreover, multiple-access neuronal communication channel is analyzed in $[10,11]$.

Neurons generate spikes in response to the external stimulus and the information is considered to be encoded in different parameters of the spike train. Some authors have exploited spike rate coding where information is encoded in the number of spikes per unit time [10] or the number of spiking bursts per unit time[12]. Another group suggests

\footnotetext{
${ }^{*}$ Corresponding author

Email address: tkhan15@ku.edu.tr(Tooba Khan), oba21@cam.ac.uk (Ozgur B. Akan)
} 


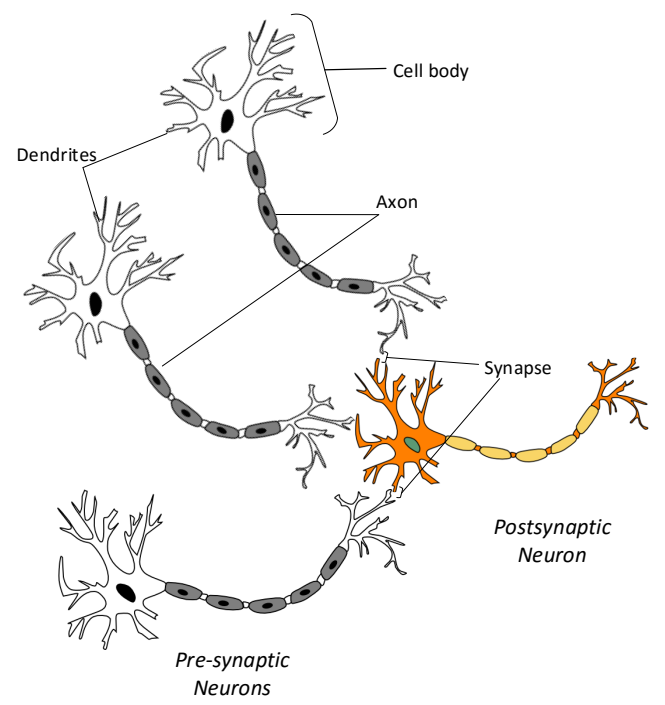

Figure 1: MISO Neuro-spike Communication Channel.

temporal coding, i.e., the information may be hidden in the exact timing of the spike [13] or in the time interval between any two spikes [14]. Information theory provides a mean to quantify information transmission between any two nodes of a communication system, therefore, it has been utilized to analyze the information transmission between neurons [15]. Single-input single-output (SISO) synaptic communication has been studied from the information and communication theoretical perspective using different channel models. The information transmission rate in SISO system with single and multiple synaptic terminals between two neurons is calculated in [16] and [17], respectively. In [18], the upper bound of the capacity for the SISO neuro-spike communication is derived where diffusion of the neurotransmitters is modeled with Bernoulli distribution. A closed-form equation for the capacity of the SISO synaptic communication channel is derived in [19] for a realistic time varying channel. The Capacity of axonal propagation within a single neuron is analyzed in [20]. In pyramidal neurons multiple pre-synaptic neurons contribute to fire a spike in the postsynaptic neuron [21]. Therefore, the information transfer rate for MISO neuro-spike communication is derived in [10]. Brain is one of the most expensive organs of any living organism in terms of energy consumption $[22,23]$. The generation of the spike and changes in membrane potential consume most of the energy reserved for the brain as it involves opening and closing of numerous ionic channels on the cell membrane [24]. Thus, the maximum achievable sum rate of the MISO channel is analyzed in [11] considering a realistic channel model with metabolic energy constraint. This study uses spike generation model for the postsynaptic neuron with constant spiking threshold.

It is evident from different neurological studies that the spiking threshold of cortical neurons displays large variability over time, profoundly influencing the encoding of information into a spike train [25, 26, 27]. Thus, the adaptive nature of the spiking threshold causes variation in neuronal response with respect to time, i.e., it is responsible for short-term plasticity [28]. Short-term synaptic plasticity leads to short-term changes in synaptic function, lasting from milliseconds to minutes, and regulates the moment-to-moment information flow through a neural circuit. Although in [19], a SISO time-variant channel with variable vesicle release probability has been analyzed, however, the effect of the dynamic spiking threshold on the information flow has not been addressed in any of the studies so far. Therefore, in this article we aim to quantify the variation in the mutual information with respect to time caused by the adaptive spiking threshold in MISO neuro-spike communication channel.

The brain consists of a variety of neurons having different characteristics. Thus, we need a model that incorporates dynamics of the spiking threshold into its responses as well as capable of reproducing spike response of any type of neuron. In INCF Quantitative Single-Neuron Modeling competition, participants were challenged to develop a model that fits to the spiking data from different neurons [29]. The most accurate one on the training data was the multi-timescale adaptive threshold (MAT)-model predicting $86 \%$ of the reliable spikes [26]. MAT-model is a simple integrate-and-fire model having adaptive threshold comprising of a summation of two components that increase at the occurrence of every spike and decay towards a resting value with different time constants. Therefore, in this paper, 


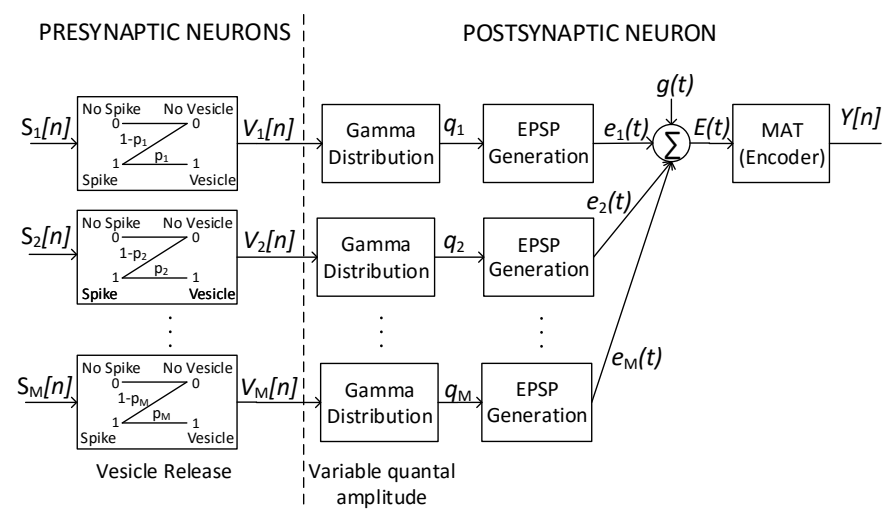

Figure 2: Block diagram of the MISO neuro-spike communication channel.

we enumerate maximum achievable sum rate of the MISO neuro-spike communication channel by integrating MATmodel as spike generation mechanism under metabolic energy constraints. Furthermore, we analyze the responses of three types of postsynaptic neurons, i.e., regular spiking (RS) neuron, intrinsic bursting (IB) neuron and fast spiking (FS) neuron, and compare their achievable rates. Moreover, we analyze the trade-off between achievable sum rates and the metabolic cost incurred, by calculating information-cost efficiency. Thus, the main objective of this study is to analyze time varying MISO neuro-spike communication channel by incorporating the effect of complex adaptive nature of the spiking threshold as well as available metabolic energy on the achievable sum rate of multiple access neuro-spike communication. Such analysis is necessary to provide the theoretical foundations on the performance of the neuronal networks before the practical implementation of bio-inspired nanonetworks. Moreover, the performance analysis of the neuronal network with different types of neurons are important in order to develop ICT-based diagnosis and treatment techniques for neurological diseases in future. Furthermore, the models of different types of neurons would enable us to select the best suited neuron type for a particular application of the bio-inspired nanonetworks based on neuron characteristics, such as, less power consumption or high encoding/transmission rates.

The rest of the paper is organized as follows. In Section 2, we describe the MISO neuro-spike communication model. In Section 3, we formulate the mutual information and sum rate of MISO neuro-spike communication channel under metabolic cost constraints. In Section 4, we present the numerical results. Finally, we conclude the paper in Section 5 .

\section{Model Description}

The basic morphology of neurons is shown in Fig. 1. The spike is generated at the soma or the cell body of a neuron and propagates through its axon to reach axonal terminal. There exists a small gap, called synapse, between any two neurons. Thus, the information transfer between neurons occurs through chemical messengers known as neurotransmitters. The axonal terminal contains vesicles, i.e., the packets of neurotransmitters, which are released in response to the spike. These neurotransmitters diffuse through the synaptic cleft to reach postsynaptic neuron [21]. The binding of neurotransmitters with the receptors present on the post-synaptic terminal opens ionic channels causing ionic influx or efflux. This ionic flow changes the post-synaptic membrane potential. If this change is greater than the threshold value, the spike is generated in the post-synaptic neuron. This study is based on a MISO neuro-spike communication channel, where a single post-synaptic neuron receives and processes information from the multiple pre-synaptic neurons as shown in Fig. 1. In this section, we define individual subprocesses of the MISO neuro-spike communication channel model shown in Fig. 2.

\subsection{Vesicle Release Process}

Each pre-synaptic neuron generates a spike train, $S_{i}(t), i \in[1, M]$, where $M$ is the number of pre-synaptic neurons. In [10] and [20], these spike trains are modeled as doubly Poisson process, i.e. , a Poisson process with a time-varying 


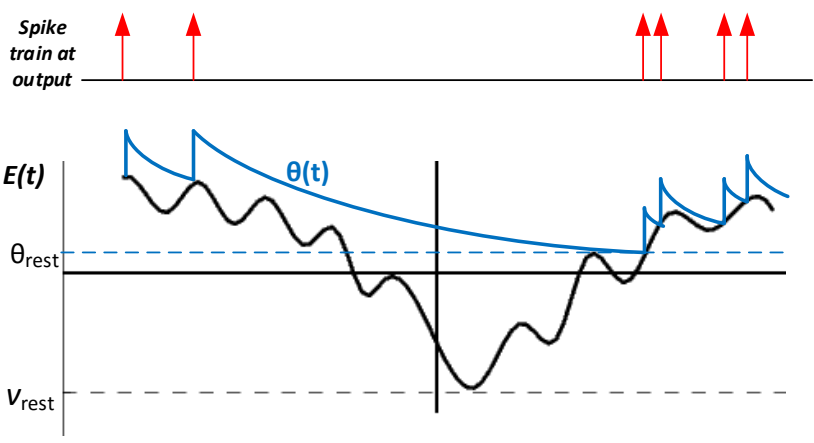

Figure 3: Spike generation with MAT model: $\theta(t)$ represents the threshold dynamics with the resting value $\theta_{\text {rest }}$ and $E(t)$ is the membrane potential with the resting value $V_{\text {rest }}$ (adapted from [26]).

spiking rate. However, to highlight the impact of the adaptive spiking threshold on the information transfer, we simplify the model by ignoring the variation in rate with time. Thus, the spike trains generated by pre-synaptic neurons are modeled by Poisson processes with constant rate $\lambda_{i}$ [30]. Few information and communication theoretical studies have focused on modeling the memory and signal transmission errors during axonal transmission and their impact on the performance of neuro-spike communication [20, 31, 32]. However, the pyramidal neurons have myelinated axons, thus, the axonal transmission is highly reliable in these neurons when they are healthy. Thus, the axon is considered as all pass filter in this paper. Therefore, the same spike train is assumed to be received at the pre-synaptic terminal.

The spike arrival at each pre-synaptic terminal causes vesicle release, which is modeled using pool-based release and replenishment model in the literature [33]. The available vesicles in the pre-synaptic terminal are divided into at least two groups or pools depending on their distance from the membrane and their mobility. The group comprising the vesicles that are ready to be released upon the arrival of spike and are the nearest to the membrane is called readily releasable pool (RRP) with size $N_{i}$ in $i$-th pre-synaptic terminal. The remaining vesicles are in reserve pool, which are used to refill the RRP after each release. In this paper, we consider the instantaneous replenishment of the ready pool same as in [10], thus, $N_{i}$ does not change with time.

We divide time in windows of equal duration, $\Delta t$, which is small enough to have only one spike. Thus, $\Delta t$ is selected equal to refractory period, i.e., $2 \mathrm{~ms}$ [26], which is the minimum allowed time interval between two consecutive spikes. During refractory period neuron does not fire even if the potential exceeds the threshold. Thus, the probability of vesicle release from $i$-th pre-synaptic terminal during $n$-th window is derived based on pool-based release model [30] as

$$
P\left\{V_{i}[n]=1 \mid S_{i}[n]=1\right\}=1-\exp \left(-\alpha_{i} N_{i}\right) \triangleq p_{i},
$$

where $\alpha_{i}=0.06 \sqrt{N_{i}}$ is the rate at which vesicles are fused with the membrane to get released [34], $S_{i}[n]=1$ and $V_{i}[n]=1$ indicate the spike arrival and vesicle release, respectively.

\subsection{Postsynaptic response at each synapse}

After each release, the post-synaptic potential caused by $i$-th synapse is modeled as [10]

$$
e_{i}(t)= \begin{cases}q_{i} h_{i} \frac{t}{t_{p}} \exp \left(1-\frac{t}{t_{p}}\right), & \text { if } t \geq 0 \\ 0, & \text { if } t<0\end{cases}
$$

where $t_{p}$ is the time to reach the peak amplitude, $h_{i}$ is the peak EPSP amplitude generated at $i$-th synapse and $q_{i}$ introduces the trial to trial variability in the magnitude of EPSP at each synapse due to random variations in the synaptic parameters such as the number of neurotransmitters per vesicle, number of neurotransmitters reaching postsynaptic membrane, number and state of available receptors and binding probability of receptors. In this paper, $q_{i}$ is modeled as Gamma random variable as in [10]. 


\subsection{Spike Generation}

The total membrane potential $E(t)$ at post-synaptic neuron contributed by all the inputs is given as

$$
E(t)=v_{\text {rest }}+\sum_{i=1}^{M} \sum_{t_{n} \leq t, \forall n} V_{i}[n] e_{i}\left(t-t_{n}\right)+g(t),
$$

where $v_{\text {rest }}$ is the membrane resting potential, $t_{n}$ is the beginning of $n$-th time window and $g(t)$ is the synaptic noise modeled by Gaussian distribution with zero mean and variance $\sigma_{n}^{2}$ [10]. The inner sum in (3) accounts for the temporal summation of the membrane potential at individual synapses and the outer sum gives the spatial summation of membrane potential across all synapses. A spike occurs in the post-synaptic neuron, i.e., $Y[n]=1$, when $E(t)$ reaches spiking threshold called $\theta(t)$, i.e., $E(t) \geq \theta(t)$ for $(n-1) \Delta t \leq t<\Delta t$.

In this paper, we use the spike generation model developed in [26] called multiple-timescale adaptive threshold (MAT) model. This model offers low computational cost as compared to other nonlinear complex biophysical models for spike generation. In adaptive threshold models, the threshold is not assumed constant since it has been observed to be varied with time [27]. Therefore, in MAT model, a dynamic threshold, i.e., $\theta(t)$, is considered that increases when spike occurs and then decays exponentially with multiple timescales towards the resting value $\theta_{\text {rest }}$ as shown in Fig. 3. The adaptive threshold, $\theta(t)$, is given as

$$
\theta(t)=\sum_{k} H\left(t-t_{k}\right)+\theta_{\text {rest }},
$$

where

$$
H(t)=\sum_{j=1}^{L} \beta_{j} \exp \left(-t / \tau_{j}\right),
$$

where $t_{k}$ is the time for generation of $k$-th spike in post-synaptic neuron, $L$ is the number of timescales or exponential functions, $\tau_{j}$ is the $j$-th time constant for exponential function, $\beta_{j}$ is the weight of the $j$-th exponential function and $\theta_{\text {rest }}$ is the resting value for the threshold. In [26], these parameters were optimized to maximize the degree of coincidence of a model spike train with a real spike train.

As apparent from its name, MAT model utilizes multiple timescales, i.e., multiple $\tau_{j}$ 's to predict the spike timings. Thus, in (4), $\theta(t)$ decays exponentially with $L$ time constants. In [26], $\tau_{j}$ are selected from 10,50 and $200 \mathrm{~ms}$ and the optimal performance was achieved with the combination of two timescales, i.e., $\mathrm{L}=2$, with $\tau_{1}=10 \mathrm{~ms}$ and $\tau_{2}=200$ ms. The performance of the MAT model found to be superior than the standard models like HH, LIF and SRM models in predicting the spike timings with the score of $0.89 \pm 0.21$.

\section{Rate Region Analysis with Metabolic Energy constraint on neuronal signaling}

In this section, we analyze information transmission rate of the MISO neuro-spike communication channel. The input spike generated at the $i$-th pre-synaptic neuron is defined as $S_{i}[n]$ and the output spike generated by a post-synaptic neuron is denoted as $Y[n]$ in the $n$-th time slot. Thus, the mutual information between $S^{M}[n]=$ $\left\{S_{1}[n], S_{2}[n], \ldots, S_{M}[n]\right\}$, as input, and $Y[n]$ can be written as

$$
I\left(S^{M}[n] ; Y[n]\right)=H(Y[n])-H\left(Y[n] \mid S^{M}[n]\right) .
$$

The total capacity of the MISO channel is the $I\left(S^{M}[n] ; Y[n]\right)$ maximized over all input distribution, $p\left(S^{M}[n]\right)$. Since we are considering the spike generation in all pre-synaptic neurons based on Poisson distribution, the mutual information can be maximized over $\lambda_{i}$ for $\forall i$ to find the maximum achievable sum rate. Defining $R_{i}[n]$ as the achievable rate for the $i$-th pre-synaptic neuron in $n$-th window, the upper bound on the achievable sum rate of the MISO channel is derived as

$$
\sum_{i=1}^{M} R_{i}[n] \leq \max _{\forall i, \lambda_{i}} I\left(S^{M}[n] ; Y[n]\right) \triangleq C[n] .
$$




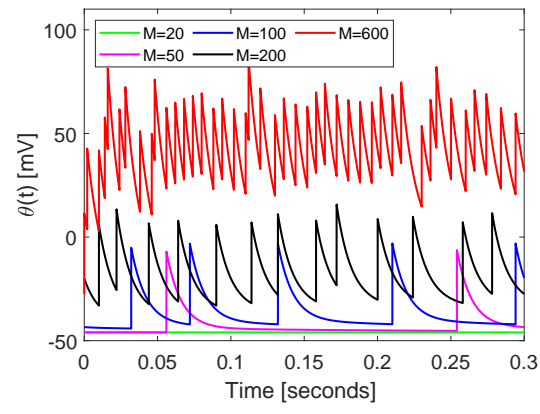

(a) Regular spiking neurons.

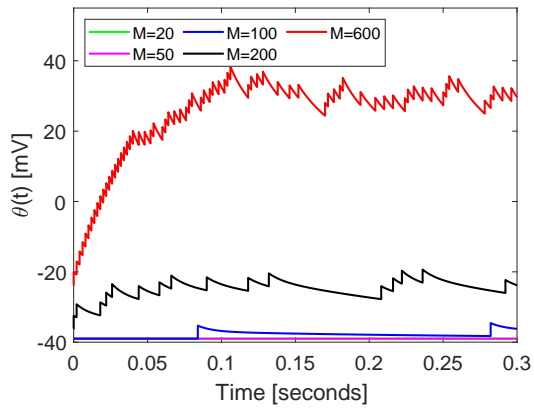

(b) Intrinsically bursting neurons.

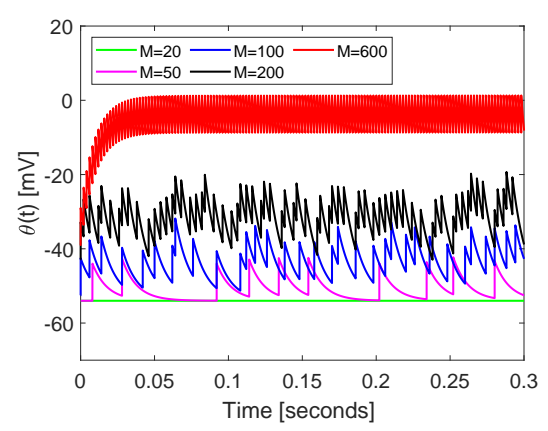

(c) Fast spiking neurons.

Figure 4: Threshold dynamics of different types of postsynaptic neurons for spiking rate, $\lambda=100$, and different number of pre-synaptic neurons, $M$.

Since the spiking threshold is changing over time, the mutual information, thus, $C[n]$, would change in each time step. Therefore, the maximum achievable sum rate of the time-varying MISO channel is defined as

$$
C=\lim _{n \rightarrow \infty} \max _{\forall i, \lambda_{i}} \sum_{l=1}^{n} \frac{I\left(S^{M}[l] ; Y[l]\right)}{n},
$$

where $n$ increases with the number of time slots. However, in neuro-spike communication channel the achievable rate is limited by the metabolic cost of neural activities, in terms of number of ATP molecules used. Major portion of these ATP molecules are used for maintaining the resting membrane potential as well as spike generation. For the given MISO channel with $M$ pre-synaptic and one post-synaptic neuron, the metabolic energy consumed in the $n$-th time interval can be calculated as [24],

$$
w\left(S^{M}[n], Y[n]\right)=(M+1) \beta \Delta t+\kappa\left(Y[n]+\sum_{i=1}^{M} S_{i}[n]\right),
$$

where $\beta=0.342 \times 10^{9}$ ATP molecules per second required to maintain resting potential and $\kappa=0.71 \times 10^{9}$ ATP molecules required to generate a single spike [35]. Thus, the average metabolic energy consumed during the $n$-th time step is

$$
w_{p}[n]=\sum_{S^{M}, Y} w\left(S^{M}[n], Y[n]\right) p\left(S^{M}[n], Y[n]\right) .
$$

According to the energy consumption explained above, the maximum achievable rate of the MISO channel would become a function of maximum possible value of $w_{p}[n]$, i.e, $W$, hence,

$$
C(W)=\lim _{n \rightarrow \infty} \max _{\substack{\forall i: \lambda_{i}, \forall l \in[1, n]: w_{p}[l] \leq W}} \sum_{l=1}^{n} \frac{I\left(S^{M}[l] ; Y[l]\right)}{n} .
$$

\section{Simulation Results and Discussion}

In this section, we simulate the MISO neuro-spike communication channel model described in Section 2 with the numerical data available in the literature as given in Table 1 . We consider $M$ pre-synaptic neurons terminating at a single post-synaptic neuron. We generate spike train in each pre-synaptic neuron using Poisson distribution with the same rate for all inputs, i.e, $\lambda_{i}=\lambda$. 


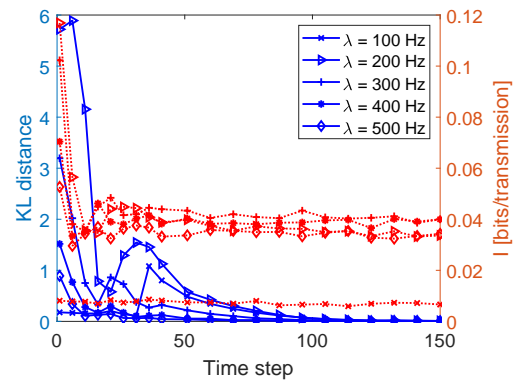

(a) Regular spiking neurons.

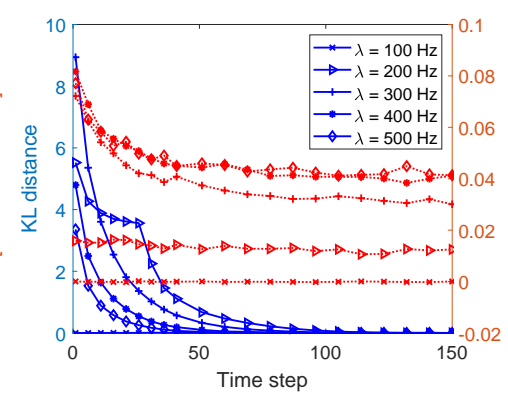

(b) Intrinsically bursting neurons.

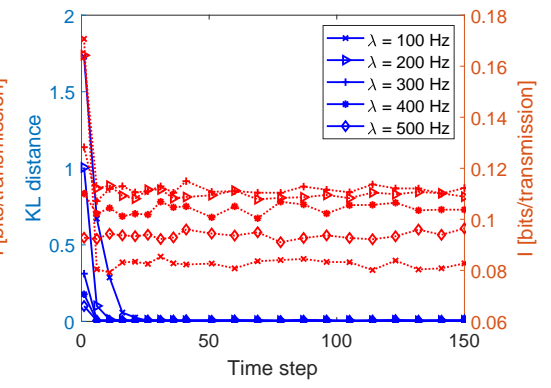

(c) Fast spiking neurons.

Figure 5: Convergence of $I\left(S^{M}[n] ; Y[n]\right)$ (dotted lines) and Kullback Leibler distances (solid lines) between the distribution of $\theta(t)$ in different time steps $(p(x))$ and the distribution in 150-th time step $(q(x))$ for $M=50$ and different spiking rates $\lambda$.

\subsection{Dynamic Spiking Threshold}

We simulate our model for three different types of postsynaptic neurons, i.e., regular spiking $(\mathrm{RS})$ neuron $\left(\theta_{\text {rest }}=\right.$ $\left.19 \mathrm{mV}, \beta_{1}=37 \mathrm{mV}, \beta_{2}=2 \mathrm{mV}\right)$, intrinsic bursting (IB) neuron $\left(\theta_{\text {rest }}=26 \mathrm{mV}, \beta_{1}=1.7 \mathrm{mV}, \beta_{2}=2 \mathrm{mV}\right)$ and fast spiking (FS) neuron $\left(\theta_{\text {rest }}=11 \mathrm{mV}, \beta_{1}=10 \mathrm{mV}, \beta_{2}=0.002 \mathrm{mV}\right)$ [26]. Note that the given values of $\theta_{\text {rest }}$ in all three cases are with reference to $v_{\text {rest }}$. The values and the number of timescales, i.e., $\tau_{j}$ and $L$, respectively, are the same for each type of neuron as mentioned in Section 2.3 and the model has been tailored to suit individual neuron by adjusting the amount of fast $\left(\tau_{1}=10 \mathrm{~ms}\right)$ and slow $\left(\tau_{2}=200 \mathrm{~ms}\right)$ dynamics, i.e., by tuning $\beta_{j}$ 's [26]. The variation in dynamic spiking threshold with respect to time is shown in Fig. 4 for different number of pre-synaptic neurons, $M$.

According to (4), the spiking threshold rises whenever spike is generated and decays back towards the resting value. It is shown in Fig. 4(c) that FS neuron can produce high number of output spikes with a very few inputs due to lower resting threshold and fast decay of the threshold. However, in IB neuron, resting value is very high and the decay is very slow as compared to RS and FS neurons, thus, it takes longer to produce spike with fewer inputs. However, as the number of inputs increases, the number of output spikes also increases approaching the behavior of FS neuron. Moreover, as can be observed in Fig. 4(a), the RS neuron maintains regular spiking behavior over a large range of inputs for the given input spiking rate, $\lambda$. However, RS behavior will also approach FS neuron with further increase in number of inputs or for higher values of lambda since it increases the probability of input spike.

Table 1: Simulation Parameters

\begin{tabular}{|l|l|l|}
\hline Parameters & Symbols & Values \\
\hline Normalized amplitude of EPSP & $h_{i}$ for $\forall i$ & $1 \mathrm{mV} \mathrm{[10]}$ \\
\hline Time to reach peak of EPSP & $t_{p}$ & $3.85 \mu \mathrm{s}[9]$ \\
\hline Size of RRP & $N_{i}$ for $\forall i$ & $10[36]$ \\
\hline Number of Threshold time constants & $L$ & $2[26]$ \\
\hline Threshold time constants & $\tau_{1}, \tau_{2}$ & $10,200 \mathrm{~ms}[26]$ \\
\hline Weights of Threshold exponents & $\alpha_{1}, \alpha_{2}$ & $37,2 \mathrm{mV} \mathrm{[26]}$ \\
\hline Resting Potential & $v_{\text {rest }}$ & $-65 \mathrm{mV}$ \\
\hline Resting threshold $\left(\right.$ w.r.t $\left.v_{\text {rest }}\right)$ & $\theta_{\text {rest }}$ & $19 \mathrm{mV} \mathrm{[26]}$ \\
\hline Mean and variance of $q_{i}$ & $\bar{q}, \sigma_{q}^{2}$ & $1,0.6[10]$ \\
\hline Noise standard deviation & $\sigma_{n}$ & $0.1 \mathrm{mV} \mathrm{[10]}$ \\
\hline
\end{tabular}




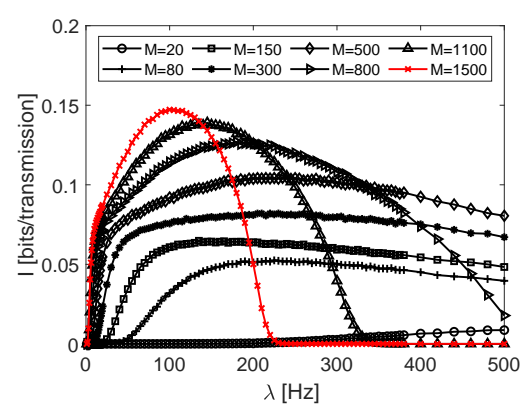

(a) Regular spiking neurons.

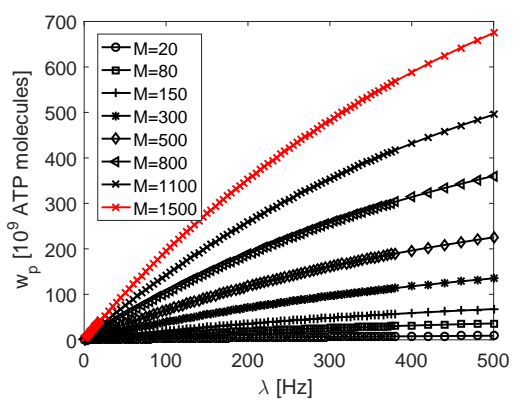

(d) Regular spiking neurons.

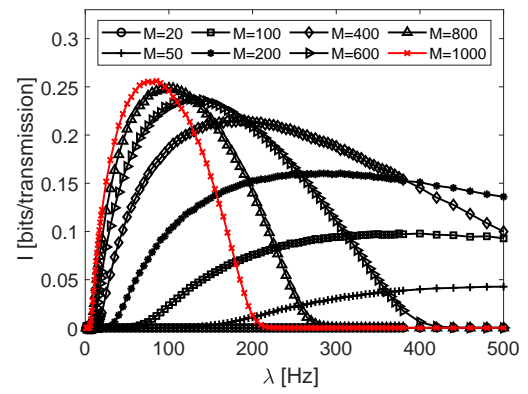

(b) Intrinsically bursting neurons.

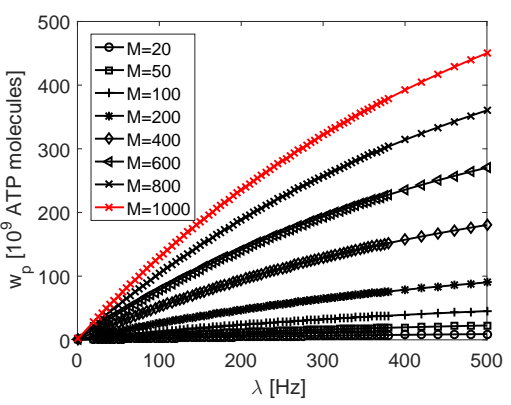

(e) Intrinsically bursting neurons.

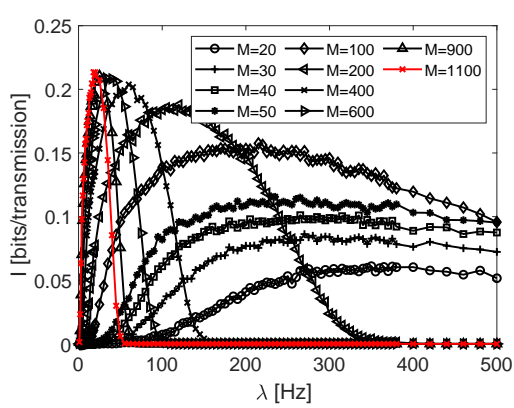

(c) Fast spiking neurons.

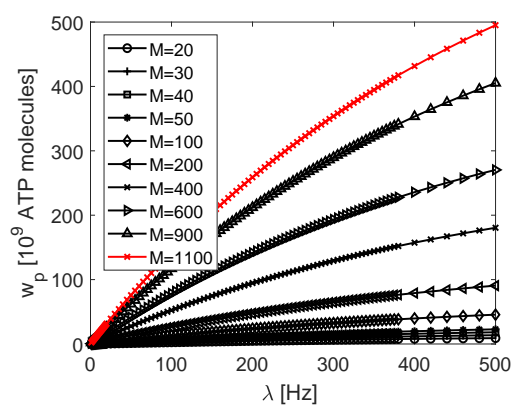

(f) Fast spiking neurons.

Figure 6: (a-c) Mutual information, $I$, and (d-f) average metabolic cost, $w_{p}$, for different number of pre-synaptic neurons, M, and spiking rates, $\lambda$.

\subsection{Mutual Information}

We simulate our model to find mutual information between $S^{M}[n]$ and $Y[n]$. Since we are using MAT-model for the spike generation, the spiking threshold, $\theta(t)$, thus, the mutual information between input and output, changes over time. To evaluate how the distribution of the spiking threshold changes over time, we use Kullback Leibler distance (KL distance) given as

$$
K L \text { distance }=\int p(x) \log _{2} \frac{p(x)}{q(x)} d x+\int q(x) \log _{2} \frac{q(x)}{p(x)} d x,
$$

where $p(x)$ and $q(x)$ are two distributions and the KL distance shows the distance between them. In Fig. 5, the change in the distribution of $\theta(t)$ for different types of neurons with $M=50$ is shown using the KL distance. Here, the $p(x)$ is the distribution of $\theta(t)$ in different time steps and $q(x)$ is the distribution in 150-th time step. We can observe that the distribution of $\theta(t)$ is converging to its steady state value in less than 150 time steps for all types of the postsynaptic neurons. Thus, we can conclude that the mutual information also converges to its steady state in the same time. To verify this conclusion, the mutual information $I\left(S^{M}[n] ; Y[n]\right)$ is also plotted (dotted lines) in Fig. 5 against time steps where its convergence can be observed. Therefore, to calculate the maximum achievable sum rate of the MISO channel, we can maximize the mutual information after reaching the steady state instead of utilizing (8). Hence, we calculate mutual information between $S^{M}[n]$ and $Y[n]$ after significant time has passed allowing the mutual information to converge for each value of $M$ and maximize it over $\lambda$ to find the maximum achievable sum rate of the MISO channel.

The mutual information, $I$, between input and output of the channel is shown in Fig. 6(a)-(c) for RS, IB and FS neurons, respectively. Increase in either spiking rate, $\lambda$, or the number of inputs, $M$, increases the number of spikes at the input, thus increasing th probability of output spike, which in turn increases the mutual information until reaching its maximum value as shown in Fig. 6(a)-(c). Moreover, this maximum mutual information is achieved with higher $\lambda$ as $M$ decreases since the reduction in $M$ reduces the number of spikes at the input, which is compensated by higher $\lambda$. We can also observe that after reaching the maximum mutual information, increasing $\lambda$ decreases $I$ since it causes more number of input spikes, which in turn decreases the chance of receiving 0 at the output, i.e., decreasing the 


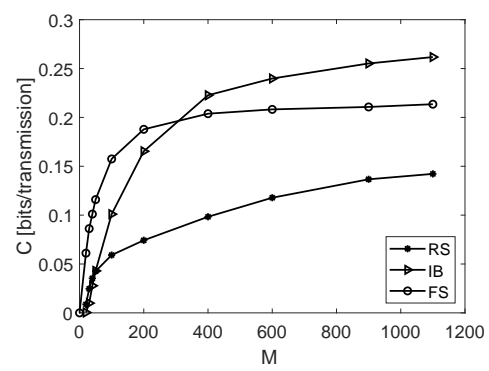

Figure 7: Maximum achievable sum rate for MISO neuro-spike communication channel for different number of input neurons.

uncertainty in the output. This argument is true for the values of $M$ as well since increasing $M$ also contributes in increase in the number of input spikes. This observation can be made in Fig. 6 for any value of $\lambda$.

The mutual information is not only dependent on input spiking rate and the number of inputs $M$, but is also affected by the inherent characteristics of a neuron. Thus, different types of the postsynaptic neurons exhibit different amount of variation in $I$ in various ranges of $M$. For instance, it can be observed from Fig. 6(b) and 6(c) that the FS Neuron shows large variation in maximum mutual information for $M<50$, however, for RS neuron, the variation in not significant for $M<50$. Thus, different simulation scenarios are considered for different types of neurons to show the variation in mutual information with respect to $M$. By comparing the three types of neurons, we notice that IB neurons achieves highest value of maximum mutual information for the given number of input neurons, closely followed by FS neurons. Moreover, we observe that for lower values of $M$, maximum mutual information is higher in FS neurons since its resting threshold is lower than the IB neurons.

The average metabolic cost, $w_{p}$, for different spiking rates, $\lambda$, and number of input neurons, $M$, is shown in Fig. 6(d)-(f). Since either increasing $\lambda$ or $M$ increases the number of spikes at the input, it increases the average metabolic energy consumed, $w_{p}$, based on (10) in either type of neurons. Moreover, since $w_{p}$ includes energy consumption by input spikes and all types of postsynaptic neurons are receiving same inputs, thus, for any given number of inputs, $w_{p}$ is similar for all types of postsynaptic neurons as presence or absence of one output spike would not affect the energy consumption significantly.

\subsection{Maximum achievable sum rate versus number of pre-synaptic inputs}

In this section, we maximize the mutual information, shown in Fig. 6(a)-(c), over $\lambda$ to estimate the maximum achievable sum rate of the MISO channel without applying the metabolic cost constraint. The relation between maximum achievable rate and the number of pre-synaptic inputs is shown in Fig. 7. It is observed that the maximum achievable sum rate, $C$, increases with increasing number of inputs, i.e., $M$, since the average number of spikes in the input increases. However, after certain value of $M$, the maximum achievable sum rate of the channel saturates, i.e, increasing the number of pre-synaptic inputs does not increase information being transmitted.

By comparing the maximum achievable sum rate of MISO channel with the three types of postsynaptic neurons in Fig. 7, we can thus conclude that IB neurons are achieving highest value of maximum achievable sum rate, $C$, for the given number of input neurons, $M$. Although the rate of increase in $C$ is higher in FS neurons for lower values of $M$. However, for $M>400$, the mutual information in FS neurons saturates while in IB neurons it continues to increase with a bit higher rate. The rate of increase of $C$ in RS neurons is slower for all values of $M$ as compared to the other neurons, thus, we can conclude that maximum achievable sum rate of MISO channel with RS neuron acting as postsynaptic neuron will saturate at a lower value than the other two neurons.

\subsection{Maximum achievable sum rate versus metabolic cost}

To enumerate the maximum achievable sum rate of the multiple access channel under metabolic cost constraint, we simulate our model for different number of pre-synaptic neurons and the results are shown in Fig. 8(a)-(c). The dotted lines in this figure show the maximum achievable sum rate that can be achieved for a particular number of inputs in the absence of metabolic cost constraint. 


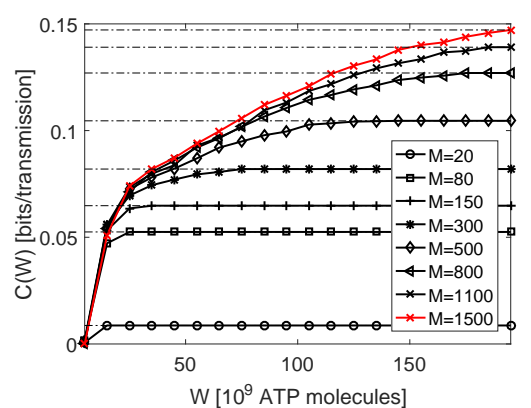

(a) Regular spiking neurons.

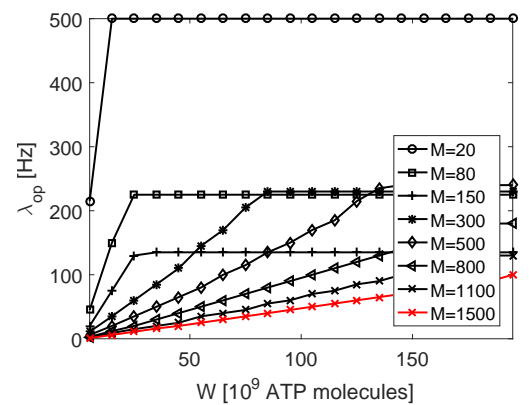

(d) Regular spiking neurons.

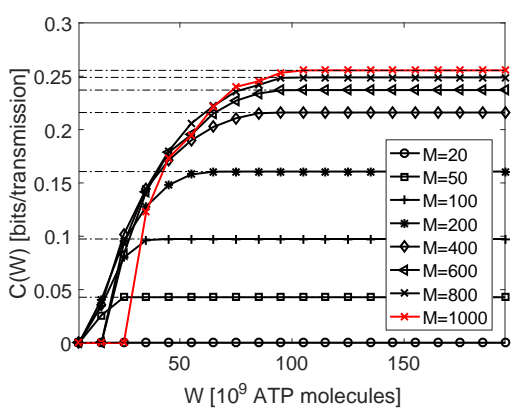

(b) Intrinsically bursting neurons.

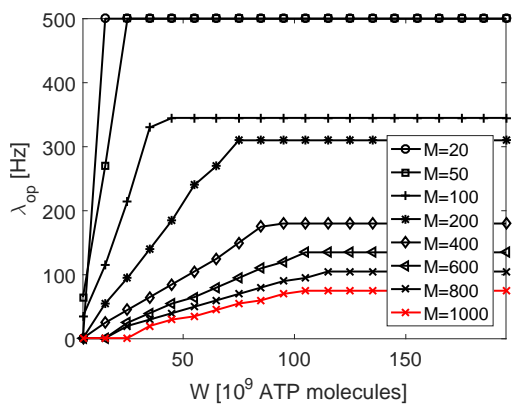

(e) Intrinsically bursting neurons.

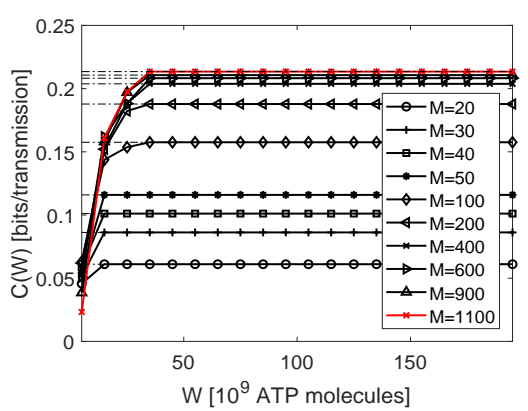

(c) Fast spiking neurons.

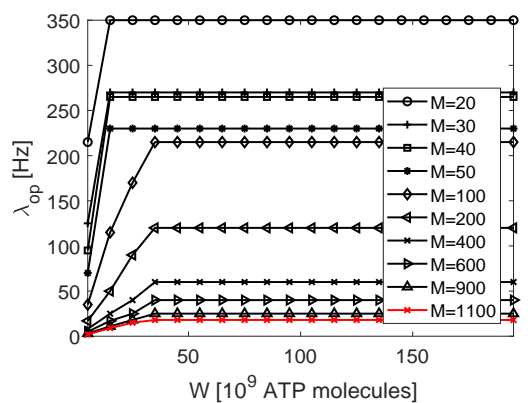

(f) Fast spiking neurons.

Figure 8: (a-c) Maximum achievable sum rate for MISO neuro-spike communication channel with metabolic cost constraint, $C(W)$, and (d-f) the spiking rate that maximizes the sum rate, $\lambda_{o p}$.

By increasing available metabolic energy, $W$, more number of spikes are allowed at the input while satisfying the metabolic cost constraint. Moreover, in Fig. 6, we observed that increasing the spiking rate at the input, i.e., number of spikes at the input, increases the mutual information. Hence, as shown in Fig. 8(a)-(c), the maximum achievable sum rate of the channel increases as we relax the upper bound on the average metabolic cost, i.e., $W$. Moreover, the maximum achievable sum rate, $C(W)$, increases with the same rate for each $M$ since $C(W)$ is a function of average number of spikes allowed at the input, which is not affected by varying number of pre-synaptic neurons, $M$, but is limited by a given value of metabolic energy, $W$. However, $C(W)$ saturates after the maximum mutual information is achieved for each value of $M$.

Enforcing metabolic cost constraint, i.e., $w_{p}<W$, limits the possible values of spiking rate, $\lambda$, used to calculate sum rate in Fig. 8. The values of $\lambda$ that achieve maximum sum rate of the MISO channel, i.e., $\lambda_{o p}$, are shown in Fig. 8(d)-(f) for different amounts of metabolic energy, $W$. For each $M$, we can observe that higher $\lambda_{o p}$ is obtained for more available energy since it allows occurrence of more spikes at the input. However, $\lambda_{o p}$ for any value of $M$ does not increase as soon as maximum mutual information is achieved. Moreover, we can also observe in Fig. 8(d)-(f) that maximum sum rate is achieved at lower $\lambda_{o p}$ for higher values of $M$ since it increases the average number of spikes at the input for any value of $\lambda$. It is observed that maximum achievable sum rate, $C(W)$ as well as the optimum spiking rate, $\lambda_{o p}$, that achieve this sum rate, follow same pattern with respect to metabolic energy for all types of postsynaptic neurons.

Comparing these results with [11], which is based on the constant spiking threshold, we conclude that very large number of inputs are needed to generate a spike in the postsynaptic neuron with dynamic spiking threshold. Thus, very high amount of energy is being consumed to achieve only half of the maximum achievable sum rate.

\subsection{Information-Cost efficiency}

As observed in Fig. 8(a)-(c), the maximum achievable sum rate stops increasing after certain value of metabolic energy, i.e., no matter ho much energy is provided the information transmission rate would not increase. Therefore, we 


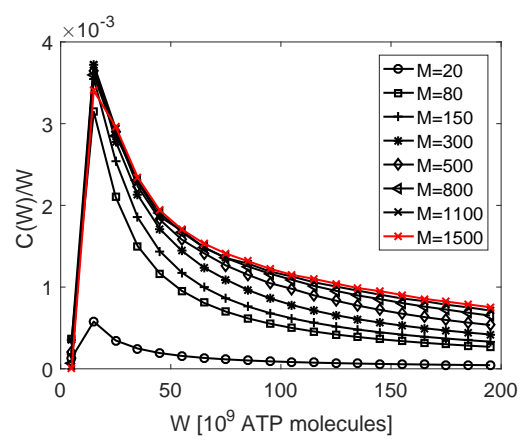

(a) Regular spiking neurons.

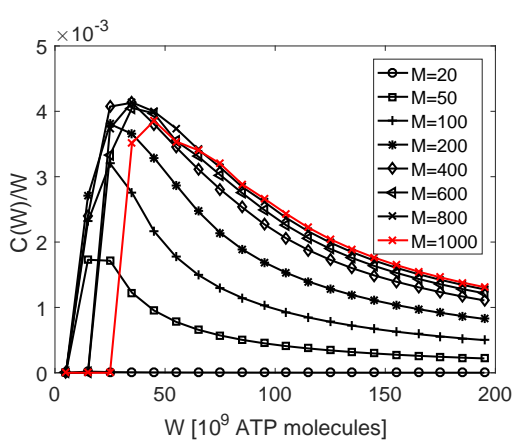

(b) Intrinsically bursting neurons.

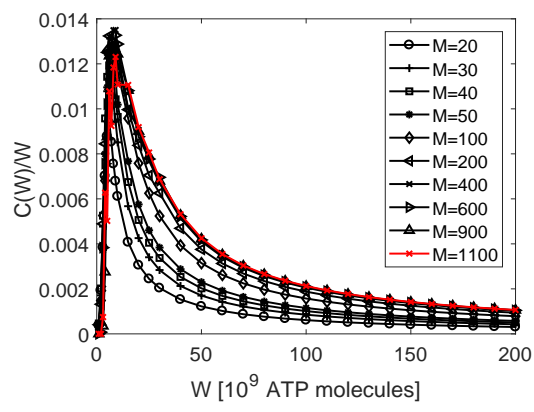

(c) Fast spiking neurons.

Figure 9: The maximum achievable sum rate per unit metabolic energy.

find a trade-off between the sum rate and the available metabolic energy, $W$, in terms of information-cost efficiency, $E=\frac{C(W)}{W}$, as shown in Fig. 9. It is observed that relaxing upper bound on $W$ beyond the optimum value would reduce the information-cost efficiency. Thus, the efficiency of the system is an important factor to optimize the energy consumption of the nanonetwork design based on MISO neuro-spike communication model.

If we compare the three types of postsynaptic neurons, we conclude that FS neuron transfers information most efficiently, i.e., it achieves the similar information transfer rate as IB neuron, however, consuming far less amount of energy. It can be observed in Fig. 8(b) and (c) that for IB neuron, maximum sum rate of $\approx 0.26$ bits/transmission is achieved for $\approx 105 \times 10^{9}$ ATP molecules and on the other hand for FS neuron, maximum sum rate of $\approx 0.22$ bits/transmission is achieved for $\approx 35 \times 10^{9}$ ATP molecules. This makes FS neurons $67 \%$ more efficient than the IB neurons.

\section{Conclusion}

Neurons use spikes to encode information form the outside world to be processed inside the brain. The cortical neurons receive inputs from the multiple pre-synaptic neurons and generate a spike when its membrane potential reaches a spiking threshold. The researchers succeeded in regenerating the spike trains considering the adaptive spiking threshold that depends on previous spike generation. Adaptive threshold is also an important factor in causing short-term plasticity in the brain, which is used to control the information flow between neurons. Thus, in this paper, we analyzed information transmission in the multiple-access neuro-spike communication channel with dynamic spiking threshold model for spike generation. Furthermore, neuro-spike communication has a major constraint in terms of metabolic energy required by the brain cells to carry out their routine processing. Therefore, we quantified the maximum achievable sum rate taking into account the metabolic cost in terms of ATP molecules consumed. Moreover, we analyzed different types of cortical neurons, i.e., RS, IB and FS neurons and concluded that FS neurons are the most efficient in terms of energy consumption, however, IB neurons provide highest data transmission rates. This analysis would help us to select the neuron with suitable characteristics for a particular application of the nanonetworks.

\section{Acknowledgements}

This work was supported in part by ERC Project MINERVA (ERC-2013-CoG 616922) and and the ERC Proof of Concept project MINRGRACE (ERC-2017-PoC 780645).

\section{References}

[1] I. F. Akyildiz, F. Brunetti, C. Blázquez, Nanonetworks: A new communication paradigm, Computer Networks 52 (12) (2008) $2260-2279$.

[2] J. M. Jornet, I. F. Akyildiz, Channel modeling and capacity analysis for electromagnetic wireless nanonetworks in the terahertz band, IEEE Transactions on Wireless Communications 10 (10) (2011) 3211-3221. 
[3] B. Atakan, O. B. Akan, S. Balasubramaniam, Body area nanonetworks with molecular communications in nanomedicine, IEEE Communications Magazine 50 (1) (2012) 28-34.

[4] O. B. Akan, H. Ramezani, T. Khan, N. A. Abbasi, M. Kuscu, Fundamentals of molecular information and communication science, Proc. of the IEEE 105 (2) (2017) 306-318.

[5] T. Nakano, M. J. Moore, F. Wei, A. V. Vasilakos, J. Shuai, Molecular communication and networking: Opportunities and challenges, IEEE trans. on nanobioscience 11 (2) (2012) 135-148.

[6] M. Pierobon, I. F. Akyildiz, A physical end-to-end model for molecular communication in nanonetworks, IEEE Journal on Selected Areas in Communications 28 (4) (2010) 602-611.

[7] L. P. Giné, I. F. Akyildiz, Molecular communication options for long range nanonetworks, Computer Networks 53 (16) (2009) $2753-2766$.

[8] E. Balevi, O. B. Akan, A physical channel model for nanoscale neuro-spike communications, IEEE Trans. on Comm. 61 (3) (2013) 11781187.

[9] T. Khan, B. A. Bilgin, O. B. Akan, Diffusion-based model for synaptic molecular communication channel, IEEE Transactions on NanoBioscience 16 (4) (2017) 299-308.

[10] D. Malak, O. B. Akan, A communication theoretical analysis of synaptic multiple-access channel in hippocampal-cortical neurons, IEEE Trans. on comm. 61 (6) (2013) 2457-2467.

[11] H. Ramezani, T. Khan, O. B. Akan, Sum rate of miso neuro-spike communication channel with constant spiking threshold, IEEE Transactions on NanoBioscience 17 (3) (2018) 342-351.

[12] K. D. Longden, M. Wicklein, B. J. Hardcastle, S. J. Huston, H. G. Krapp, Spike burst coding of translatory optic flow and depth from motion in the fly visual system, Current Biology 27 (21) (2017) 3225-3236.

[13] K. Aghababaiyan, V. Shah-Mansouri, B. Maham, Asynchronous neuro-spike array-based communication, in: 2018 IEEE International Black Sea Conference on Communications and Networking (BlackSeaCom), IEEE, 2018, pp. 1-5.

[14] K. Aghababaiyan, V. Shah-Mansouri, B. Maham, Capacity bounds of neuro-spike communication by exploiting temporal modulations, in: Wireless Communications and Networking Conference (WCNC), 2018 IEEE, IEEE, 2018, pp. 1-6.

[15] A. Borst, F. E. Theunissen, Information theory and neural coding, Nature neuroscience 2 (11) (1999) $947-957$.

[16] A. Manwani, C. Koch, Detecting and estimating signals over noisy and unreliable synapses: information-theoretic analysis, Neural computation 13 (1) (2001) 1-33.

[17] H. Ramezani, C. Koca, O. B. Akan, Rate region analysis of multi-terminal neuronal nanoscale molecular communication channel, in: Proc. of IEEE Nano, 2017.

[18] M. Veletić, P. A. Floor, Y. Chahibi, I. Balasingham, On the upper bound of the information capacity in neuronal synapses, IEEE Trans Commun. 64 (12) (2016) 5025-5036.

[19] H. Ramezani, T. khan, O. B. Akan, Information theoretical analysis of synaptic communication for nanonetworks, in: Proc. 37th IEEE INFOCOM, IEEE, 2018.

[20] K. Aghababaiyan, V. Shah-Mansouri, B. Maham, Axonal channel capacity in neuro-spike communication, IEEE transactions on nanobioscience 17 (1) (2018) 78-87.

[21] M. F. Bear, B. W. Connors, M. A. Paradiso, Neuroscience: Exploring the Brain, 3rd Edition, Lippincott Williams \& Wilkins, 2007.

[22] S. B. Laughlin, T. J. Sejnowski, Communication in neuronal networks, Science 301 (5641) (2003) 1870-1874.

[23] B. Sengupta, S. B. Laughlin, J. E. Niven, Balanced excitatory and inhibitory synaptic currents promote efficient coding and metabolic efficiency, PLoS Comput Biol 9 (10) (2013) e1003263.

[24] D. Attwell, S. B. Laughlin, An energy budget for signaling in the grey matter of the brain, Journal of Cerebral Blood Flow \& Metabolism 21 (10) (2001) 1133-1145.

[25] J. Platkiewicz, R. Brette, A threshold equation for action potential initiation, PLoS Comput Biol 6 (7) (2010) e1000850.

[26] R. Kobayashi, Y. Tsubo, S. Shinomoto, Made-to-order spiking neuron model equipped with a multi-timescale adaptive threshold, Front. in comp. neuroscience 3 (2009) 9.

[27] B. Fontaine, J. L. Peña, R. Brette, Spike-threshold adaptation predicted by membrane potential dynamics in vivo, PLoS Comput Biol 10 (4) (2014) e1003560.

[28] J. F. Mejias, J. J. Torres, Emergence of resonances in neural systems: the interplay between adaptive threshold and short-term synaptic plasticity, PloS one 6 (3) (2011) e17255.

[29] C. Rossant, D. F. Goodman, B. Fontaine, J. Platkiewicz, A. K. Magnusson, R. Brette, Fitting neuron models to spike trains, Frontiers in neuroscience 5 (2011) 9.

[30] H. Ramezani, O. B. Akan, Information capacity of vesicle release in neuro-spike communication, IEEE Communications Letters 22 (1) (2017) 41-44.

[31] H. Ramezani, O. B. Akan, A communication theoretical modeling of axonal propagation in hippocampal pyramidal neurons, IEEE transactions on nanobioscience 16 (4) (2017) 248-256.

[32] H. Ramezani, O. B. Akan, Impacts of spike shape variations on synaptic communication, IEEE Transactions on NanoBioscience 17 (3) (2018) 260-271.

[33] V. Matveev, X.-J. Wang, Implications of all-or-none synaptic transmission and short-term depression beyond vesicle depletion: a computational study, J. Neurosci. 20 (4) (2000) 1575-1588.

[34] L. E. Dobrunz, C. F. Stevens, Heterogeneity of release probability, facilitation, and depletion at central synapses, Neuron 18 (6) (1997) 995-1008.

[35] L. Kostal, R. Kobayashi, Optimal decoding and information transmission in hodgkin-huxley neurons under metabolic cost constraints, Biosys. $136(2015) 3-10$

[36] T. Schikorski, C. F. Stevens, Quantitative ultrastructural analysis of hippocampal excitatory synapses, J. Neurosci. 17 (15) (1997) $5858-5867$. 